\title{
An artificial meteor database as a test for the presence of weak showers
}

\author{
A. Olech ${ }^{1}$ and M. Wiśniewski ${ }^{2}$ \\ 1 Nicolaus Copernicus Astronomical Center, ul. Bartycka 18, 00-716 Warszawa, Poland \\ e-mail: olech@camk.edu.pl \\ 2 Warsaw University Observatory Al.Ujazdowskie 4, 00-478 Warszawa, Poland \\ e-mail: mwisniew@sirius .astrouw.edu.pl \\ Received 22 October 2001 / Accepted 7 January 2002
}

\begin{abstract}
We have constructed an artificial meteor database resembling in all details the real sample collected by the observers of the Comets and Meteors Workshop in the years 1996-1999. The artificial database includes the sporadic meteors and also events from the following showers: Perseids, Aquarid complex, $\alpha$-Capricornids, July Pegasids and Sagittarids. This database was searched for the presence of the radiants of two weak showers: $\alpha$-Cygnids and Delphinids. The lack of these radiants in the artificial database and their existence in the real observations suggests that $\alpha$-Cygnids and Delphinids are real showers and their radiants could not be formed as an effect of intersections of back prolongated paths of meteors belonging to other showers.
\end{abstract}

Key words. solar system: general - meteors, meteoroids

\section{Introduction}

Recently, Polish observers taking part in the Comets and Meteors Workshop (CMW) reported the rediscovery of two July meteor showers - $\alpha$-Cygnids and Delphinids (Olech et al. 1999a, 1999b; Stelmach \& Olech 2000; Wiśniewski \& Olech 2000, 2001). Both of these showers are weak with maximum Zenithal Hourly Rates (ZHRs) slightly exceeding or below the sporadic background.

The $\alpha$-Cygnids are active from the end of June until the end of July. The highest activity with $Z H R=2.4 \pm$ 0.1 is observed at a solar longitude $\lambda_{\odot}=114.8 \pm 0.5^{\circ}$. The radiant of the shower at this moment is placed at $\alpha=305^{\circ}$ and $\delta=+45^{\circ}$.

The activity period of the Delphinids is still quite uncertain with the first meteors from this shower detected around July 10 and the last ones as late as the middle of August. According to the recent work of Wiśniewski \& Olech (2001) the maximum hourly rates are observed at $\lambda_{\odot}=125.0 \pm 0.1^{\circ}$. The activity at this moment is equal to $Z H R=2.2 \pm 0.2$ and the radiant of the shower has the equatorial coordinates equal to $\alpha=312^{\circ}$ and $\delta=+12^{\circ}$.

The equatorial coordinates of the beginnings and ends of meteor paths and the angular velocities for both showers were carefully analyzed using the RADIANT software (Arlt 1992). This software takes into account the properties of the observed meteors and computes the maps of

Send offprint requests to: A. Olech, e-mail: olech@camk.edu.pl probability for the presence of a radiant (hereafter PPR maps).

Although PPR maps computed for both of these showers showed distinct features, the resulting radiants were polluted by the influence of the meteors from other showers. A quite strong tail reaching the radiant of the Perseids was detected in the case of the $\alpha$-Cygnids. There is also a trace of the weak $o$-Draconids radiant in the close vicinity of the radiant of the $\alpha$-Cygnids (Olech et al. in preparation).

An even more complicated situation is present in the case of the Delphinids. The radiant of this shower is placed not far from the series of ecliptic radiants of the Aquarids complex, $\alpha$-Capricornids and the Sagittarids.

The radiants of these showers are large and have a complex structure often showing several maxima of activity.

Thus one can suspect that both the $\alpha$-Cygnids and Delphinids are not real showers and their radiants produced by RADIANT software come from crossing the backprolongated paths of the meteors from other showers active in July and also from sporadic events.

To check this possibility, we decided to construct a realistic database of artificial meteors which thoroughly resembled the real sample analyzed in the above mentioned papers.

\section{July showers}

There are many meteor showers active in July. The most active of them are the Perseids and $\delta$-Aquarids $\mathrm{S}$ but there 
Table 1. VMDB statistics for each July shower from years 1996-1999.

\begin{tabular}{|l|c|c|c|c|}
\hline \hline Shower & Activity period & $N_{\text {met }}$ & $N_{\text {spor }}$ & $T_{\text {eff }}$ \\
\hline \hline Sagittarids & Jul. 1-15 & 302 & 3072 & 400.6 \\
July Pegasids & Jul. 5-15 & 649 & 5237 & 612.8 \\
$\alpha$-Capricor. & Jul. 1-Aug. 25 & 5403 & 68735 & 7469.3 \\
$\delta$-Aquarids N & Jul. 10-Aug. 28 & 4091 & 48782 & 5244.9 \\
$\delta$-Aquarids S & Jul. 6-Aug. 22 & 3841 & 28355 & 2746.6 \\
$\iota$-Aquarids S & Jul. 14-Aug. 25 & 1241 & 25808 & 2404.3 \\
Perseids & Jul. 13-Aug. 5 & 13953 & 38060 & 3937.2 \\
\hline \hline
\end{tabular}

are also several minor showers such as the Sagittarids, the July Pegasids, $\alpha$-Capricornids, $\delta$-Aquarids $\mathrm{N}$ and the ı-Aquarids N (Rendtel et al. 1995).

Recent compilations of meteor shower activity were done by Jenniskens (1994) and Rendtel et al. (1995). The first of these papers presented the results obtained in the years 1981-1991 and the second the results from the period 1988-1995. On the other hand, the databases used by Olech et al. (1999a, 1999b), Stelmach \& Olech (2000) and Wiśniewski \& Olech (2000, 2001) were obtained using observations collected in the years 1996-1999. Because this period does not overlap with those mentioned above we decided to make a new compilation of meteor activity in July.

We used the Visual Meteor Databases (VMDB) constructed each year by the International Meteor Organization (IMO) and accessible via the IMO web pages (Arlt 1997, 1998, 1999, 2000).

The observations from the databases were selected according to the following criteria:

- data with limiting magnitudes less than 5.5 are omitted;

- observing intervals should be longer than 0.5 hour;

- radiant altitude should be at least 20 degrees;

- field of view has less than $50 \%$ cloud obstruction.

The numbers of meteors from each shower, the numbers of sporadic events, the activity periods used for computation of activity profiles and the effective time of observations in these periods are summarized in Table 1.

Following the example of Jenniskens (1994) we fitted the following equation format to each activity profile:

$$
Z H R=Z H R_{\max } \cdot 10^{-B\left|\lambda_{\odot}-\lambda_{\odot}^{\max }\right|}
$$

where $\lambda_{\odot}$ denotes the solar longitude for the epoch of 2000.0, and Zenithal Hourly Rates (ZHRs) are computed as follows:

$$
Z H R=\frac{N \cdot F \cdot r^{(6.5-L M)}}{T_{\mathrm{eff}} \cdot \sin \left(H_{\mathrm{rad}}\right)}
$$

where $N$ is a number of meteors observed during $T_{\text {eff }}, F$ is the cloud correction factor, $H_{\mathrm{rad}}$ is the altitude of the radiant, $L M$ is the limiting magnitude in the field of view and $r$ is the population index, which values are taken from Rendtel et al. (1995).
Table 2. $Z H R_{\max }, \lambda_{\odot}^{\max }, B$ parameters and assumed radiant radii $R$ for July showers.

\begin{tabular}{|l|r|r|r|c|}
\hline \hline Shower & $Z H R_{\max }$ & $\lambda_{\odot}^{\max }\left[{ }^{\circ}\right]$ & $B\left[1 /^{\circ}\right]$ & $R\left[^{\circ}\right]$ \\
\hline \hline July Pegasids & 3.11 & 108.52 & 0.0760 & 0.8 \\
& \pm 0.13 & \pm 0.24 & \pm 0.0101 & \\
\hline$\alpha$-Capricornids & 3.41 & 126.23 & 0.0352 & 2.5 \\
& \pm 0.05 & \pm 0.17 & \pm 0.0008 & \\
\hline$\delta$-Aquarids N & 2.62 & 130.03 & 0.0213 & 1.0 \\
& \pm 0.05 & \pm 0.33 & \pm 0.0009 & \\
\hline$\delta$-Aquarids S & 8.99 & 127.05 & 0.0666 & 1.0 \\
& \pm 0.16 & \pm 0.11 & \pm 0.0013 & \\
\hline -Aquarids S & 2.49 & 126.92 & 0.0491 & 1.0 \\
& \pm 0.08 & \pm 0.27 & \pm 0.0020 & \\
\hline Sagittarids & 3.00 & 90.00 & 0.0351 & 3.0 \\
& - & - & \pm 0.0015 & \\
\hline Perseids & 17.0 & 136.70 & 0.0430 & 0.8 \\
& - & - & \pm 0.0010 & \\
\hline \hline
\end{tabular}

For each of the analyzed showers we computed $Z H R-$ $\lambda_{\odot}$ dependence and we fitted it using the formula given in Eq. (1). Free parameters $Z H R_{\max }, \lambda_{\odot}^{\max }$ and $B$ were determined using the least squares method.

The results obtained for all July showers are presented in Table 2 and their activity profiles drawn using the Eq. (1) shown in Fig. 1.

Two showers from our sample required special treatment. As was shown by Jenniskens (1994) in the case of the Perseids, a nearly exponential increase in activity is observed only between $\lambda_{\odot}=120^{\circ}$ and $\lambda_{\odot}=137^{\circ}$ with $B=0.050 \pm 0.005$ and $Z H R$ at the end of this period is equal to 18 . After $\lambda_{\odot}=137^{\circ}$ the slope $B$ changes to $0.20 \pm 0.01$, and after $\lambda_{\odot}=141.8^{\circ}$ changes again to $0.083 \pm 0.017$. Thus, we cannot describe the activity of the Perseids using only one formula in the form of (1). Fortunately we are interested only in the Perseids activity in July and therefore we analyzed data for this stream only for $\lambda_{\odot}<137^{\circ}$. For this shower we obtained that at $\lambda_{\odot}=136.7^{\circ}$ the $Z H R_{\max }$ is equal to 17.0. Thus in the case of the Perseids, the only free parameter in the Eq. (1) is $B$.

Another unusual shower is the Sagittarids for which Rendtel et al. (1995) found several maxima of activity. In this case, we computed the $Z H R$ for the beginning of July and assumed this moment as the maximum. Thus again the only free parameter was the slope $B$.

A completely different approach we performed in the case of sporadic meteors. As was shown in Znojil (1995) the hourly rates of sporadic events increase almost linearly between 22 and 2 at local time with a slope equal to 2.1 meteors per hour. Using VMDB of IMO we also computed the mean value of $H R$ for sporadic meteors in July. Thus the sporadic background for each night was described by the following equation:

$H R_{\text {spor }}=2.12 \cdot \mathrm{UT}-33.64$. 


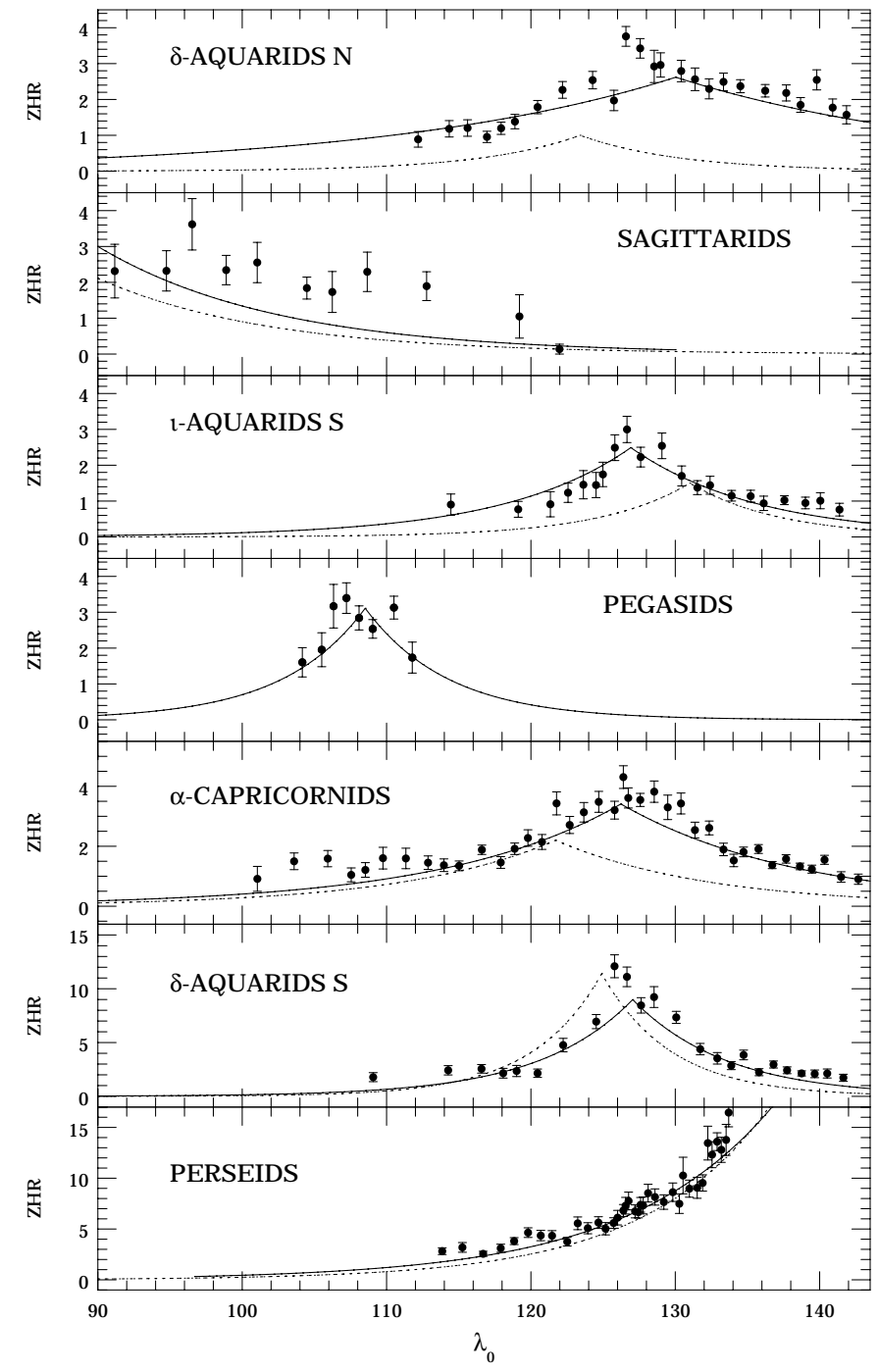

Fig. 1. Activity profiles for July showers. The solid lines correspond to the fits based on the Eq. (1). The dotted lines are the activity profiles given by Jenniskens (1994).

\section{Observations}

The sample analyzed by Wiśniewski \& Olech (2001) contained 1372 hours of effective time of observations collected in 1996-1999. Due to the poor weather conditions in Poland these observations were not distributed uniformly. Thus the artificial database, which we want to construct should take into account the distribution of real observations. This distribution is presented in Fig. 2.

Due to the fact that the majority of the $C M W$ observations were made during the astronomical camps, which took place in the Observational Station of Warsaw University Observatory in Ostrowik, we assumed that all observations in the artificial sample were collected in Ostrowik $\left(\lambda=21.4^{\circ} \mathrm{E}, \phi=52.1^{\circ} \mathrm{N}\right)$. We also assumed that the limiting magnitude $L M$ was the same for all observations and was at the level of $6.3 \mathrm{mag}$, a value typical for Ostrowik conditions.

Knowing the number of observations collected on each July night we distributed them uniformly from 20:00 to

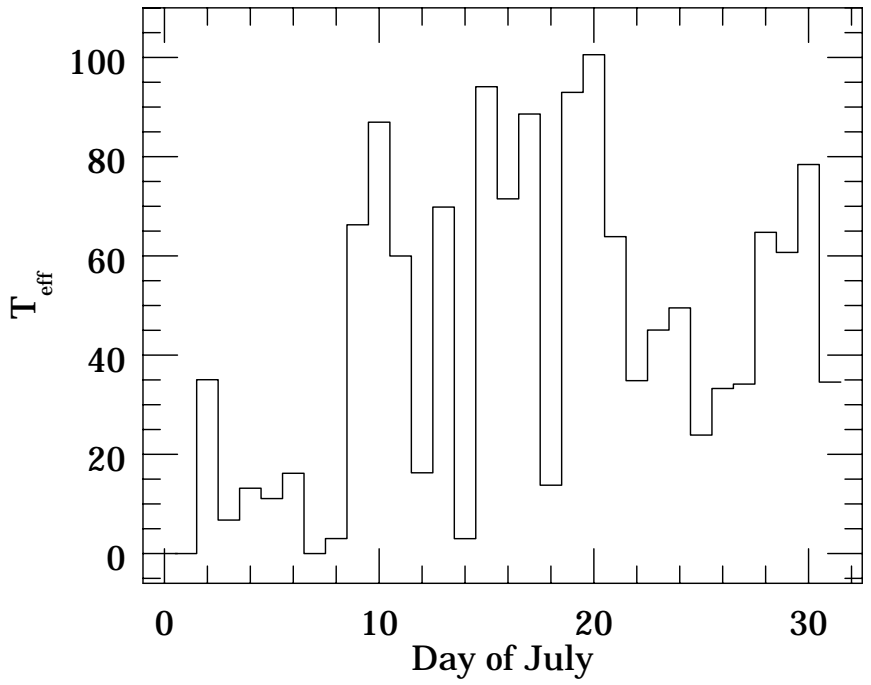

Fig. 2. Distribution of real observations collected by the $C M W$ observers in the years 1996-1999.

24:00 UT-the period of time in which the Sun is sufficiently enough below the horizon for meteor observations in Poland.

For each moment, using values from Table 2 and the Eq. (1), we can compute the expected value of $Z H R$ for each shower. Knowing the $Z H R$ s, and also the values of altitudes of the radiants for each hour, and using Eq. (2), we can calculate the expected numbers of observed meteors $N$. Of course, due to the different perception of the observers, some of them, even in the same conditions, detect a higher number of meteors than others. Thus we modified the expected numbers $N$ using Gaussian distribution with the mean at $N$ and the standard deviation equal to $\sqrt{N}$.

As a result we obtained a file containing the date of the observation, the UT time at the middle of the observation and the numbers of meteors from each shower and also the number of sporadic meteors.

Finally in our artificial sample we included $1042 \alpha$ Capricornids, $750 \delta$-Aquarids N, 605 July Pegasids, 803 $\delta$-Aquarids S, $396 \iota$-Aquarids S, 121 Sagittarids, 2465 Perseids and 15394 sporadics. This sample is clearly more numerous than the real database. It is due to the fact that during the construction of the artificial sample we assumed a constant and quite high value of the limiting magnitude $(L M=6.3 \mathrm{mag})$ and additionally assumed that all artificial observations were taken under a clear sky.

\section{Distribution of shower meteors over the celestial sphere}

\subsection{Locations of meteor paths}

Due to the small perturbations caused by the bodies of the Solar System, paths of the meteoroids from a particular stream are not ideally parallel in the Earth atmosphere. Thus the radiants of meteor showers are 
not the ideal points. According to recent video results (Molau 2000, 2001) the radiant radii of the Perseids and the Leonids are around 1 degree. For more complex ecliptic radiants like the $\alpha$-Capricornids, the Sagittarids and the Taurids, these radii are around 3 degrees (Molau 2000; Triglav 2001). In our artificial database we have to take into account these radiant sizes. Thus for showers like the Perseids and the July Pegasids, which are rich in young material, assumed radiant radii were equal to 0.8 degree. Other normal showers have these radii equal to 1 degree. For the Sagittarids and the $\alpha$-Capricornids we assumed radii equal to 3.0 and 2.5 degrees, respectively. These values are summarized in Table 2.

Knowing the theoretical radiant center and its radius, for each event from a given shower we calculated its real radiant position using the two dimensional Gaussian surfaces with a center at the theoretical radiant center and standard deviations in right ascension and declination equal to the radiant radius given in Table 2 .

During the real observation each observer of the $C M W$ was looking in a specific direction. These details are noted in observational report form by giving the equatorial coordinates of the center of the field of view. This center should be always at an elevation of at least 40 degrees.

The artificial database should take into account a location of the field of view of an observer. Therefore we constructed a list of the centers of view used in the real sample. In the first step, for each artificial observation, we randomly drew a center of the field of view from our list.

Having a center we can analyze the distribution of the meteors in the field of view. Using the real observations we calculated the distances of the beginnings of the meteor paths from the center of the field of view. The distribution of these distances is presented in Fig. 3. The best fit to this distribution was obtained using the Gaussian function with the mean value equal to $24.9^{\circ}$ and $\sigma=15.2^{\circ}$, shown as a solid line in Fig. 3.

Of course meteors appear irrespective of the distance from the field of view. Another factor which we should take into account is the location of the radiant. Thus, finding the beginning of the meteor path was done as follows:

1. knowing the center of the field of view, the distance of the beginning of the meteor path was drawn using the distribution presented in Fig. 3;

2. the line between the center of the field of view and the radiant of the shower was found;

3 . the angle between the line mentioned above and the line connecting the center of the field of view with the beginning of the meteor path was found using the Gaussian distribution with a mean equal to zero and a standard deviation equal to $60^{\circ}$;

4. to have a quite uniform distribution of meteors around the center of the field in $40 \%$ of the cases, the angle mentioned above was increased by $180^{\circ}$.

In the next step, after finding the beginning of a meteor, we computed the length of the meteor path. For this purpose we used equations given in Rendtel et al. (1995).

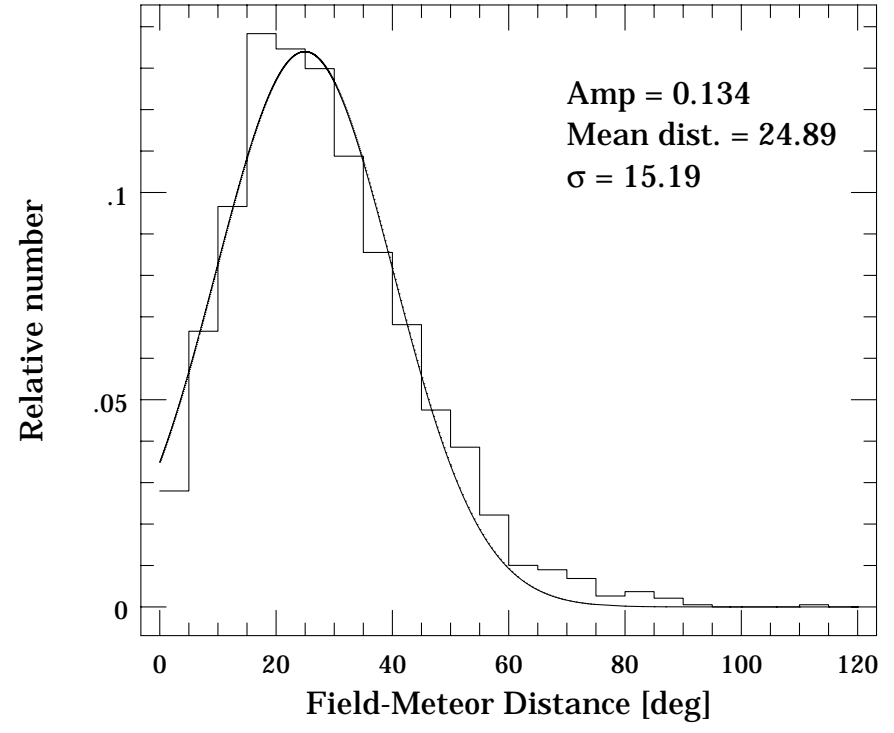

Fig. 3. The distribution of distances between the center of the observed field and the beginning of the meteor path for real data collected by the $C M W$ observers.

Knowing the radiant position, the beginning of the meteor path and its length we were able to find the equatorial coordinates of the end of the meteor.

\subsection{Introducing the errors}

During a real observation no one is capable of exactly determining the meteor's path and velocity. Thus in an artificial database we have to modify the paths and velocities of the events introducing the errors caused by the observers.

There are two error components which affect the direction and position of the meteor path. They are a tilt $\epsilon$ and a parallel shift $d$ as is shown in Fig. 4. An analysis of these quantities in visual observations made by experienced observers was done by Koschack (1991). Their distributions, according to that paper, are shown in Fig. 5. The solid lines correspond to the Gaussian fits described by the parameters given also in Fig. 5.

We used these fits for randomly drawing the tilt and shift for each meteor and modifying its path.

Due to the errors made by the observer the path of the meteor is not only tilted and shifted. Additionally observers often change the length of the meteor path, plotting it as shorter or longer in comparison with its real length.

In our approach we calculated the observed length of the meteor path $l$ and modified its beginning and end using the Gaussian distributions with a mean value equal to zero and a standard deviation equal to $l / 10$.

More than just the direction and length of the meteor are modified due to the errors inputted by the observer. Another factor which is randomly changed during 


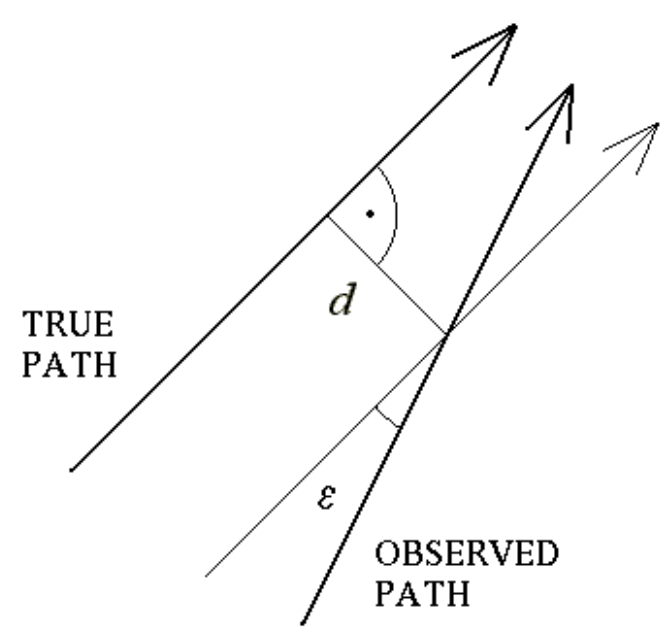

Fig. 4. The plotting errors a tilt $\epsilon$ and a parallel shift $d$.

an observation is the meteor's angular velocity. Knowing the entry velocity $V_{\infty}$ of the event and its location on the celestial sphere we can calculate its theoretical angular velocity.

As was shown by Koschack (1991) the error in angular velocity inputted by the observer depends on the angular velocity itself. The distribution of these errors in different ranges of velocities is shown in Fig. 6. Each of these distributions was fitted with a Gaussian function shown as solid line. The mean values of these Gaussian functions were assumed as zero and the obtained standard deviations are given in each panel.

These distributions were used for randomly modifying the angular velocities of the meteors in our artificial sample.

As an example of a result, in Figs. 7 and 8, we show different kinds of maps produced by RADIANT software for our sample of 2465 Perseids. Figure 7 shows results obtained for paths of the Perseids before introducing the errors made by the observer. In the upper panel of this figure we show the paths of the meteors in the sky. The map is centered at the radiant of the Perseids for $\lambda_{\odot}=$ $125^{\circ}$. The largest circle has a radius equal to $90^{\circ}$. One can note that some meteors do not radiate exactly from the center of the picture. This is caused by the fact that the radiant of the Perseids moves across the celestial sphere and meteors observed at the middle of July radiate from another point than meteors noted one or two weeks later.

In the middle panel of Fig. 7 we show an intersection map obtained using the tracings mode of the RADIANT software. Due to the assumed almost one degree radius of the radiant, not all meteors intersect in the center of the radiant. The elongated shape of the radiant is caused by the distribution of the meteors which are observed mostly at the western side of the radiant at this time of the year.

In the lower panel of Fig. 7 we show the PPR map for our artificial sample of Perseids. It is clear that the radiant is compact and circular and its position is correct.
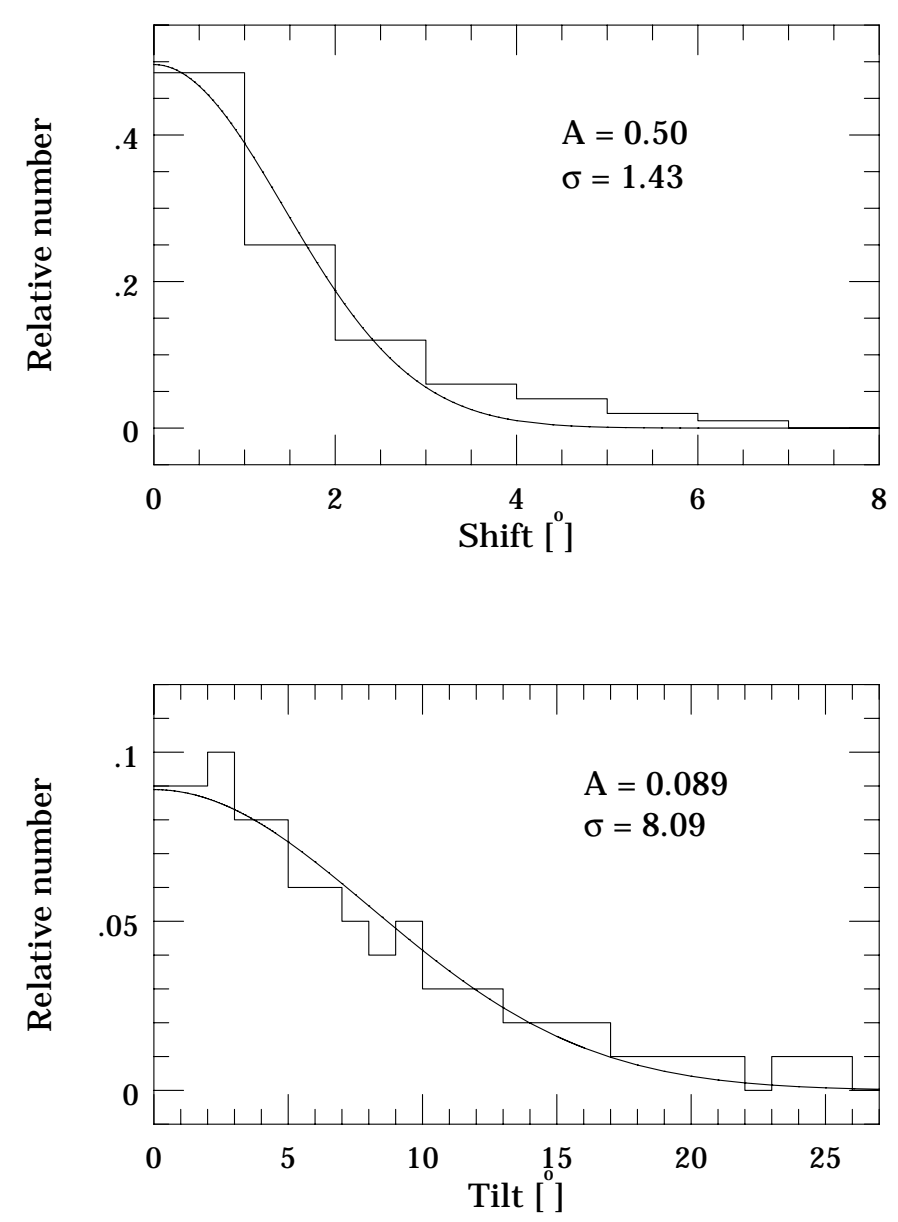

Fig. 5. The distribution of tilts and shifts obtained from observations by experienced observers according to Koschack (1991). Solid lines denote the Gaussian functions with amplitude and standard deviation given in each panel.

The same analysis was made for the Perseids with meteor paths and velocities randomly changed using the observational errors and the result is shown in three panels of Fig. 8. At the upper panel one can notice that meteors do not always radiate exactly from the center of the radiant.

Although the radiant obtained using the tracings method, shown in the middle panel, is still very clear it is also more diffuse in comparison with the middle panel of Fig. 7. It is worth noting that there is a different scale used in both middle panels. In Fig. 7 the highest number of intersections detected in the center of the radiant is 1066 and in Fig. 8 it reaches only 180.

The PPR map obtained for the sample of Perseids with introduced observational errors shows a radiant which is still circular and at the correct position but significantly more diffuse than in previous case. 


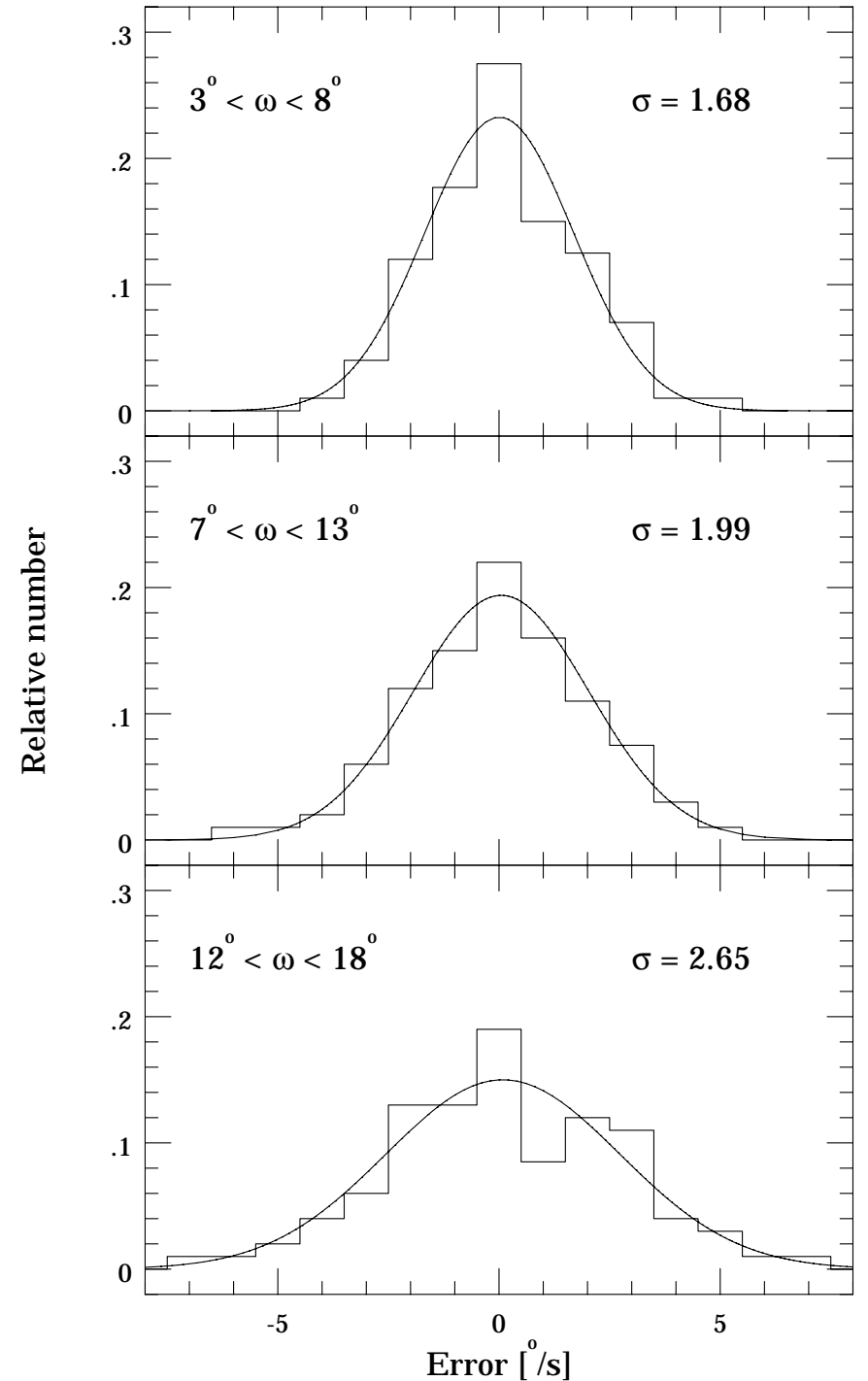

Fig. 6. The distribution of errors in angular velocities for observations of experienced observers according to Koschack (1991). Solid lines denote the Gaussian functions with a standard deviation given in each panel.

\section{Distribution of sporadic meteors over the celestial sphere}

Since the 1950 s we know that sporadic meteor radiants are not distributed uniformly over the celestial sphere, but they are concentrated in particular regions which are similar to radiants with radii slightly larger than $20^{\circ}$ and positions approximately fixed relative to the Sun. The first three sources associated with the ecliptic plane were discovered by Hawkins (1957). According to the latest papers (Jones \& Brown 1993; Brown \& Jones 1995; Poole 1997) we now identify six such sources. They are antihelion, helion, northern and southern toroidal centers and also the northern and southern apex.

When constructing the sample database of meteors we have taken into account the above mentioned sources.

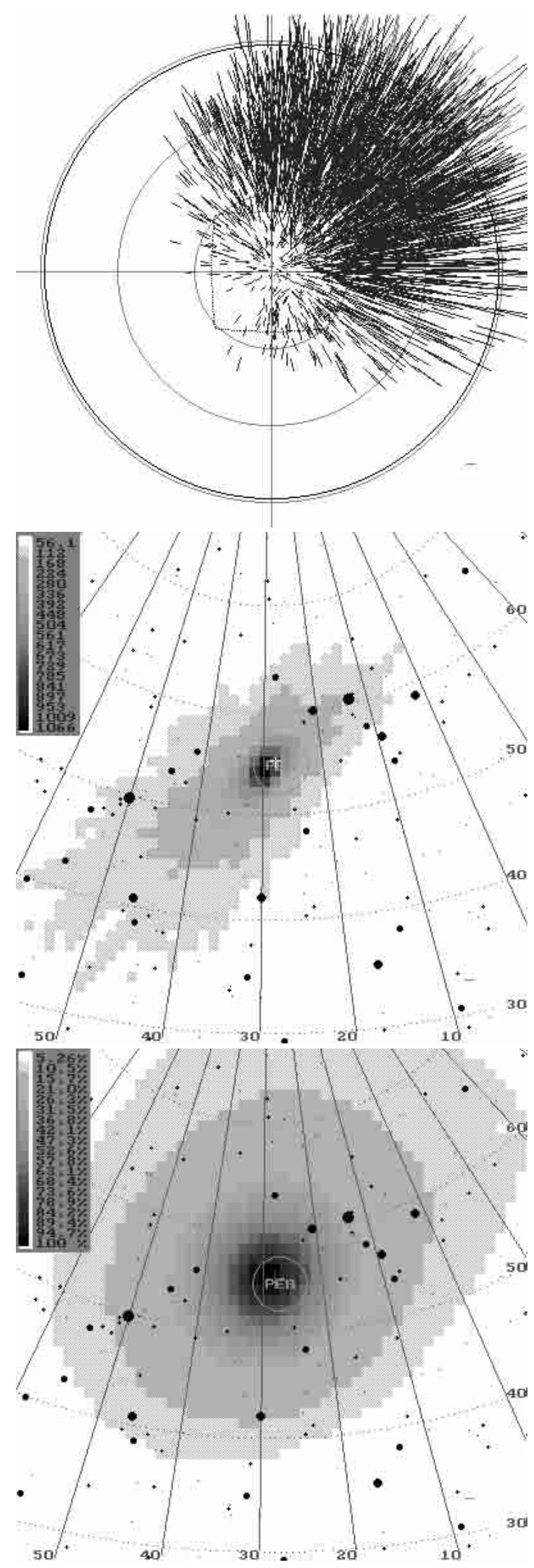

Fig. 7. Upper panel: sample distribution of the Perseid shower meteor paths (observational errors not included yet). The center of the figure is at the radiant of the Perseids. Middle panel: the radiant of the Perseids obtained using the tracings method of the RADIANT software. Lower panel: PPR map of the Perseids. 

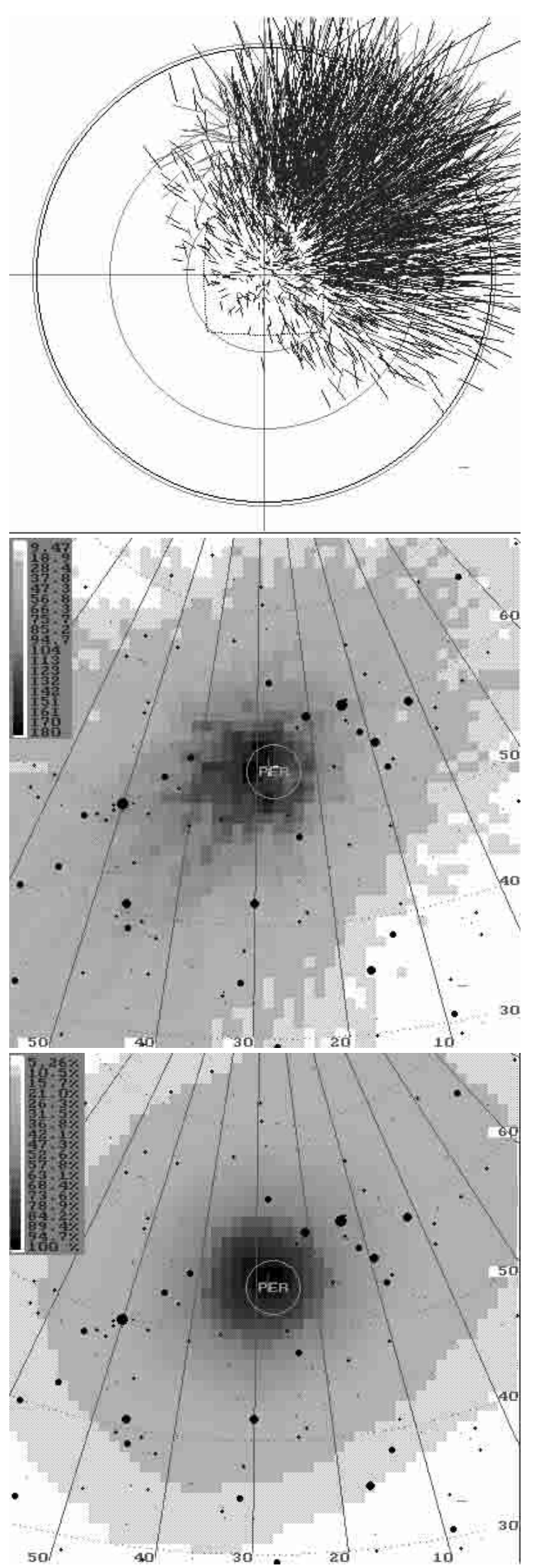

Fig. 8. Upper panel: sample distribution of the Perseid shower meteor paths with observational errors included. The center of the figure is at the radiant of the Perseids. Middle panel: the radiant of the Perseids obtained using the tracings method of the RADIANT software. Lower panel: PPR map of Perseids.
Table 3. The ecliptic coordinates and radii of the sporadic meteor sources included in our artificial database.

\begin{tabular}{|l|c|c|c|}
\hline \hline Source & $\lambda-\lambda \odot$ & $\beta$ & $R\left[^{\circ}\right]$ \\
\hline \hline Antihelion & $198^{\circ}$ & $0^{\circ}$ & $18^{\circ}$ \\
Northern Toroidal Source & $271^{\circ}$ & $+57^{\circ}$ & $19^{\circ}$ \\
Norther Apex & $271^{\circ}$ & $+19^{\circ}$ & $21^{\circ}$ \\
\hline \hline
\end{tabular}

We decided to omit the helion, southern toroidal center and the southern apex. These radiants in July at Polish geographical coordinates are always either very close to the Sun or below the horizon. The remaining three sources were included into the database and their properties taken from Jones \& Brown (1993) are summarized in Table 4.

According to the work of Jones \& Brown (1993) and Poole (1997) all sources seem to have similar activity. Thus we assumed that the number of the sporadic events radiating from each center is proportional only to the sine function of the center altitude. The whole number of sporadics observed during each hour was divided between these sources. Especially in the evening hours, when all centers are either below the horizon or only slightly over it, we assumed the existence of another source. It was centered at the zenith and its radiant had a radius equal to $60^{\circ}$.

Knowing the positions and the radii of all four sources, for the purpose of finding the location of sporadic meteor paths, we followed the procedures applied for shower meteors described in Sect. 4.1.

Also the procedures for introducing the errors into the meteor paths was the same as described in Sect. 4.2.

The sporadic sources, contrary to the shower radiants, are not characterized by meteors with a common entry velocity. Thus for each sporadic source we used the entry velocity distributions given by Jones \& Brown (1993) (see their Figs. 7-9). These velocities were also changed according to the error distributions presented in Fig. 6.

\section{6. $\alpha$-Cygnids}

The $\alpha$-Cygnid shower was discovered by W. F. Denning (1919). After his observations we have rather poor information about the activity of this shower. A reasonable determination of the position of the radiant was done using photographic observations, and based on the one captured event, the equatorial coordinates of the radiant were $\alpha=304.5^{\circ}$ and $\delta=+48.7^{\circ}$ with a geocentric velocity equal to $V_{\infty}=41.0 \mathrm{~km} \mathrm{~s}^{-1}$ (Babadzhanov \& Kramer 1961)

The first comprehensive study of the $\alpha$-Cygnids, based on the 11 years of visual observations, was presented by Jenniskens (1994). According to this work the $\alpha$-Cygnids are a weak shower with maximum $Z H R$ s equal to $2.5 \pm 0.8$ occurring at a solar longitude $\lambda_{\odot}=116.0^{\circ}$. The meteors from this shower are observed from $\lambda_{\odot}=105^{\circ}$ to $\lambda_{\odot}=127^{\circ}$. 
In recent years the $\alpha$-Cygnid shower was intensively analyzed by the Polish Comets and Meteors Workshop (Olech et al. 1999a, 1999b; Stelmach \& Olech 2000). Here we only briefly mention the results presented in the latest of these papers, which is based on the most comprehensive sample. According to Stelmach \& Olech (2000) the radiant of the $\alpha$-Cygnids is at $\alpha=305^{\circ}$ and $\delta=+45^{\circ}$. The activity of this shower lasts from the beginning to the end of July with a quite obvious maximum at $\lambda_{\odot}=114.8^{\circ} \pm 0.5^{\circ}$. Maximal $Z H R$ s are equal to $2.4 \pm 0.1$.

As was pointed out by Olech et al. (1999a) the $\alpha$ Cygnid shower was probably not recognized before the study of Jenniskens (1994) because of a lack of data in photographic meteor databases around the peak date of the shower.

Stelmach \& Olech (2000) presented only the preliminary results for year 1999. As the $C M W$ database from period 1996-1999 is complete now, we decided to recalculate PPR maps for $\alpha$-Cygnids. We selected 6772 meteors observed in period June 30-July 31. The PPR maps were centered at $\alpha=303^{\circ}$ and $\delta=+45^{\circ}$. According to earlier results, computations were performed for the moment of $\lambda_{\odot(\max )}=115^{\circ}$ with daily drift of the radiant equal to $\Delta \lambda=+1.0^{\circ}$. The assumed entry velocity was $V_{\infty}=41 \mathrm{~km} \mathrm{~s}^{-1}$. From our sample we excluded meteors slower than $1 \%$ s and faster than $30^{\circ} / \mathrm{s}$. The calculations were done rejecting meteors placed at distances larger than 50 and 85 degrees from the radiant. The final results are shown in Fig. 9.

One can clearly detect the circular radiant close to the center of each map. The RADIANT software allows two ways of estimating the radiant position from the PPR map. The first of them uses the framed part of a PPR map and computes the simple mean position of the radiant weighted by the values of computed probability. The second one also uses the framed part of a PPR map but in this case the probability distribution is fitted with a twodimensional Gaussian function. These options were used for each of our two PPR maps resulting with four radiant position determinations. The simple mean of these values is $\alpha=303.9^{\circ} \pm 0.5^{\circ}$ and $\delta=+45.3^{\circ} \pm 0.6^{\circ}$. The quoted errors are simple standard deviations of the mean and due to the fact that our four determinations are not completely independent, the real errors might be from two to three times larger.

A similar approach was undertaken using the artificial sample and the results are shown in two panels of Fig. 10, where we presented the PPR maps calculated rejecting meteors placed at distances larger than 50 and 85 degrees from the suspected position of the radiant. There is no trace of any circular structure as was detected in the case of real meteors. High probabilities (black areas) at these figures are caused by two reasons. First, the PPR map is always scaled to the highest probability point and its value is assumed to be $100 \%$. Taking into account that we assumed the diffuse sporadic source placed at a zenith with a radius equal to $60^{\circ}$, we should expect the presence of this feature in our maps. The zenith, at Polish latitudes in July,
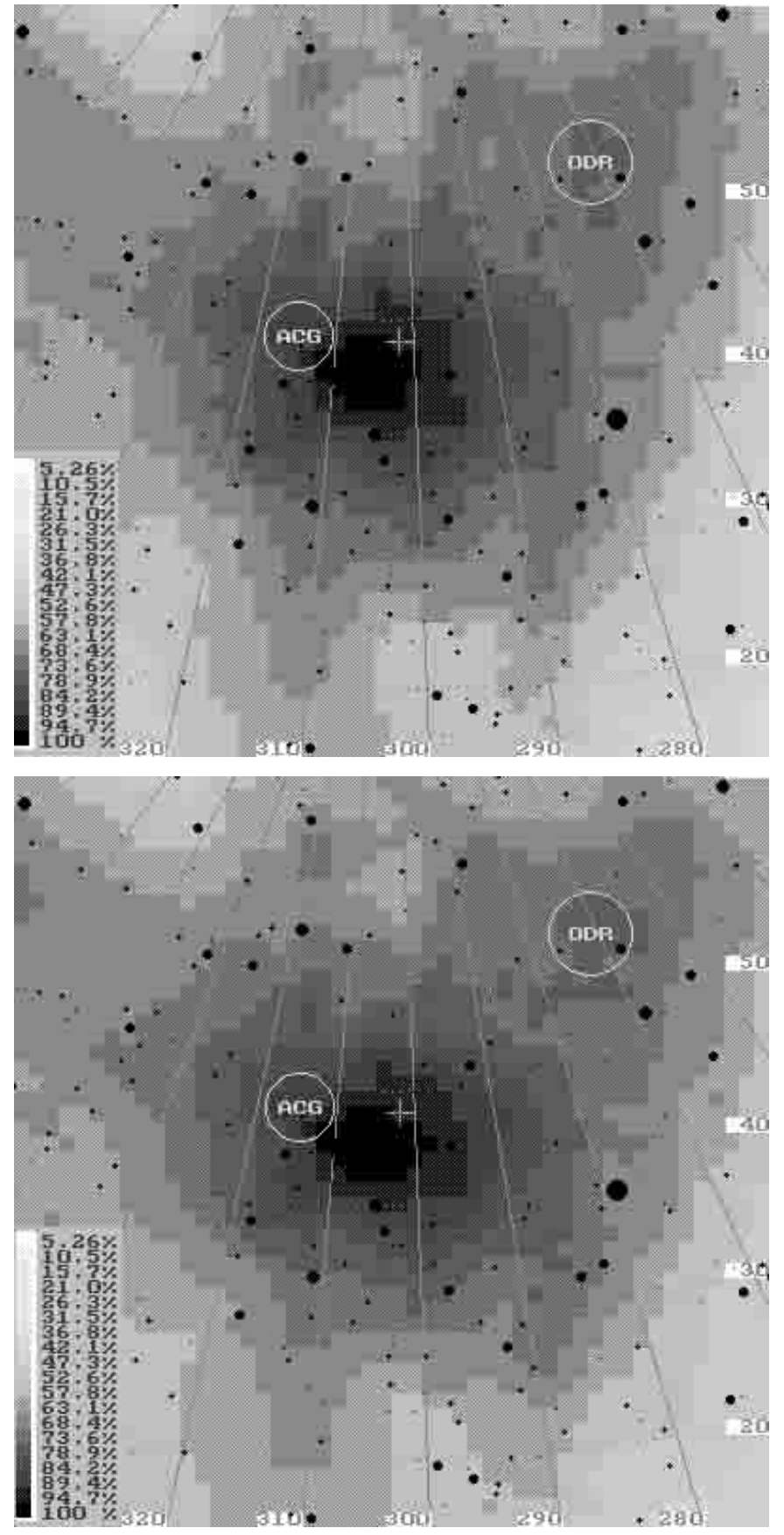

Fig. 9. PPR maps for a real sample of 6772 meteors observed in years 1996-1999. All maps are computed for the following parameters: $\lambda_{\odot}=115^{\circ}, \Delta \lambda=1.0^{\circ}$ and $V_{\infty}=41 \mathrm{~km} \mathrm{~s}^{-1}$. The maximum distance of the meteor from the radiant is 50 and $85^{\circ}$, respectively from the upper to lower panel.

lies near Deneb ( $\alpha \mathrm{Cyg}$ ) thus we are not surprised by the high probabilities of detecting the meteors radiating from this region of the sky. But, as we mentioned, the PPR map for the artificial sample does not show any circular radiant as in the case of the real sample, strongly suggesting that the $\alpha$-Cygnids are the real shower.

Our conclusions are confirmed by a comparison of the real and artificial sample maps obtained by the tracings 

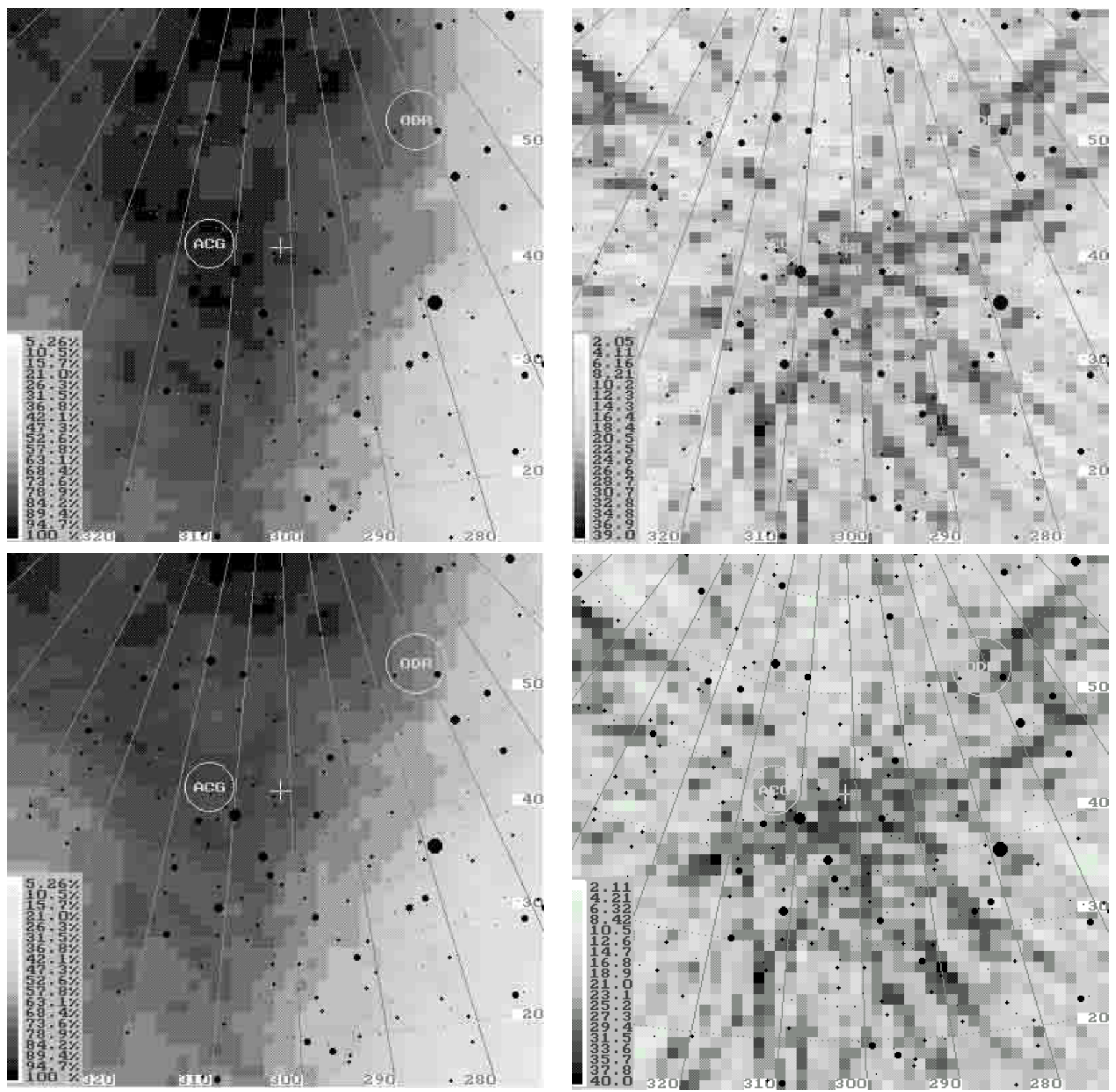

Fig. 10. PPR maps for an artificial sample of 21516 meteors. All maps are computed for the following parameters: $\lambda_{\odot}=$ $115^{\circ}, \Delta \lambda=1.0^{\circ}$ and $V_{\infty}=41 \mathrm{~km} \mathrm{~s}^{-1}$. The maximum distance of the meteor from the radiant is 50 and $85^{\circ}$, respectively from the upper to lower panel.

method of the RADIANT software. In Fig. 11 we have presented tracings maps for real sample meteors placed respectively within 50 and 85 degrees from the center of the map. In both cases one can see a clear enhancement of the intersections (about 30-40) at the radiant of the $\alpha$-Cygnids. No such picture is present in the case of the artificial sample for which the results are shown in Fig. 12. Now, the distribution of intersections is more diffuse and

Fig. 11. Tracings maps for a real sample of 6772 meteors observed in the years 1996-1999. All maps are computed for the following parameters: $\lambda_{\odot}=115^{\circ}, \Delta \lambda=1.0^{\circ}$ and $V_{\infty}=$ $41 \mathrm{~km} \mathrm{~s}^{-1}$. The maximum distance of the meteor from the radiant is 50 and $85^{\circ}$, respectively from the upper to lower panel.

is centered mostly at the zenith rather than at the center of the map.

Finally, we conclude that we are unable to reproduce the circular and high quality picture of the radiant of the $\alpha$-Cygnids using the artificial sample. Such a structure is clearly detected in the real database strongly suggesting that the $\alpha$-Cygnid shower indeed exists. 

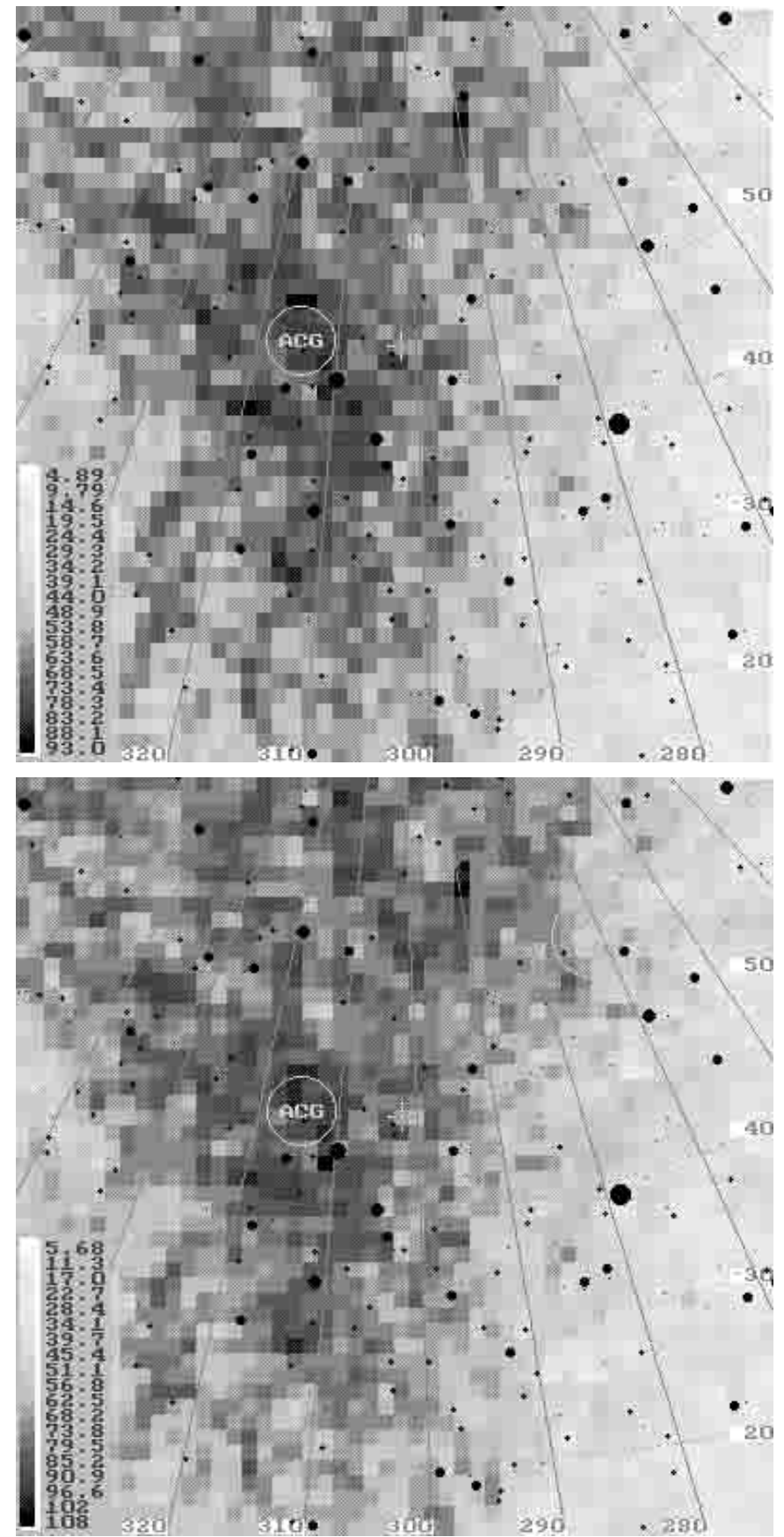

Fig. 12. Tracings maps for an artificial sample of 21516 meteors. All maps are computed for the following parameters: $\lambda_{\odot}=115^{\circ}, \Delta \lambda=1.0^{\circ}$ and $V_{\infty}=41 \mathrm{~km} \mathrm{~s}^{-1}$. The maximum distance of the meteor from the radiant is 50 and $85^{\circ}$, respectively from the upper to lower panel.

\section{Delphinids}

The Delphinids are not a new shower. The existence of meteors radiating from the constellation of Delphinus was suggested by Russian and Polish meteor sources (Abalakin 1981; Kosinski 1990). In the beginning of the 1990s the shower was studied by Bulgarian observers (Velkov 1996). The comprehensive analysis of this shower was undertaken by the Comets and Meteors Workshop
(Olech et al. 1999b; Wiśniewski \& Olech 2000, 2001). It showed that the Delphinids are a very weak shower with the maximum at $\lambda_{\odot}=125^{\circ}$ with $Z H R=2.2 \pm 0.2$.

The estimated radiant position was $\alpha=312^{\circ}$ and $\delta=+12^{\circ}$. It is very close to the antihelion source of sporadic meteors, whose position for $\lambda_{\odot}=125^{\circ}$ is $\alpha=325^{\circ}$ and $\delta=+14^{\circ}$. Thus one can presume that the radiant of the Delphinids obtained by Wiśniewski \& Olech (2000, 2001) is not real but rather comes from crossing the paths of meteors from the antihelion source and other ecliptic showers active during the second part of July.

To clarify this situation we decided to reanalyze the real sample of Wiśniewski \& Olech (2001) collected in the years 1996-1999. We selected 6468 meteors observed between July 9th and July 31st and then computed PPR maps for meteors within 50 and 85 degrees from the radiant. We also assumed that the entry velocity $V_{\infty}$ is equal to $35 \mathrm{~km} \mathrm{~s}^{-1}$ and meteors in the sky are slower than $30^{\circ} / \mathrm{s}$. The assumed daily drift of the radiant was $\Delta \lambda=1.0^{\circ}$.

The results of our computation are presented in two panels of Fig. 13. The upper panel shows the radiant of the Delphinids for meteors closer than $50^{\circ}$ from the center of the map. The big cross marks the position of the antihelion source. As in the case of the $\alpha$-Cygnids we computed the mean position of the Delphinids' radiant combining the estimates obtained for different PPR maps shown in Fig. 13. This mean position, equal to $\alpha=313.4^{\circ} \pm 0.6^{\circ}$ and $\delta=+8.6^{\circ} \pm 2.7^{\circ}$, is marked by the asterisk.

A similar computation was performed for 20376 meteors from the artificial sample. All parameters were the same as in the case of the real sample. The results are shown in Fig. 14. Again the big cross denotes the position of the antihelion source and the asterisk the radiant of the Delphinids obtained from visual observations of the $C M W$.

A comparison of Figs. 13 and 14 shows clear differences. First, in the case of the real sample, the radiant of the Delphinids is quite compact and suffers from intense pollution from ecliptic showers and the $\alpha$-Cygnids only in PPR maps shown in the lower panel. The first panel, showing only the closest meteors, gives a quite circular radiant in a clearly different position from the antihelion source. All panels obtained for the artificial sample, at the position of the radiant of the Delphinids, give the probability of detecting the radiant around $70 \%$. This is significantly lower comparing with almost $100 \%$ probability at the position of the $\delta$-Aquarids $\mathrm{N}$ radiant and also at the antihelion source. High probabilities are also detected in the northern part of all figures caused by the putting a large and diffuse radiant in the zenith.

All these PPR maps strongly suggest that Delphinids indeed exist but detecting this shower, due to the vicinity of ecliptic showers and the antihelion source, requires a large sample of good quality data.

Following the approach done for the $\alpha$-Cygnids and described in the previous paragraph, we computed the tracings maps for both real and simulated samples. The results are shown in Figs. 15 and. 16. Because the top panels, 

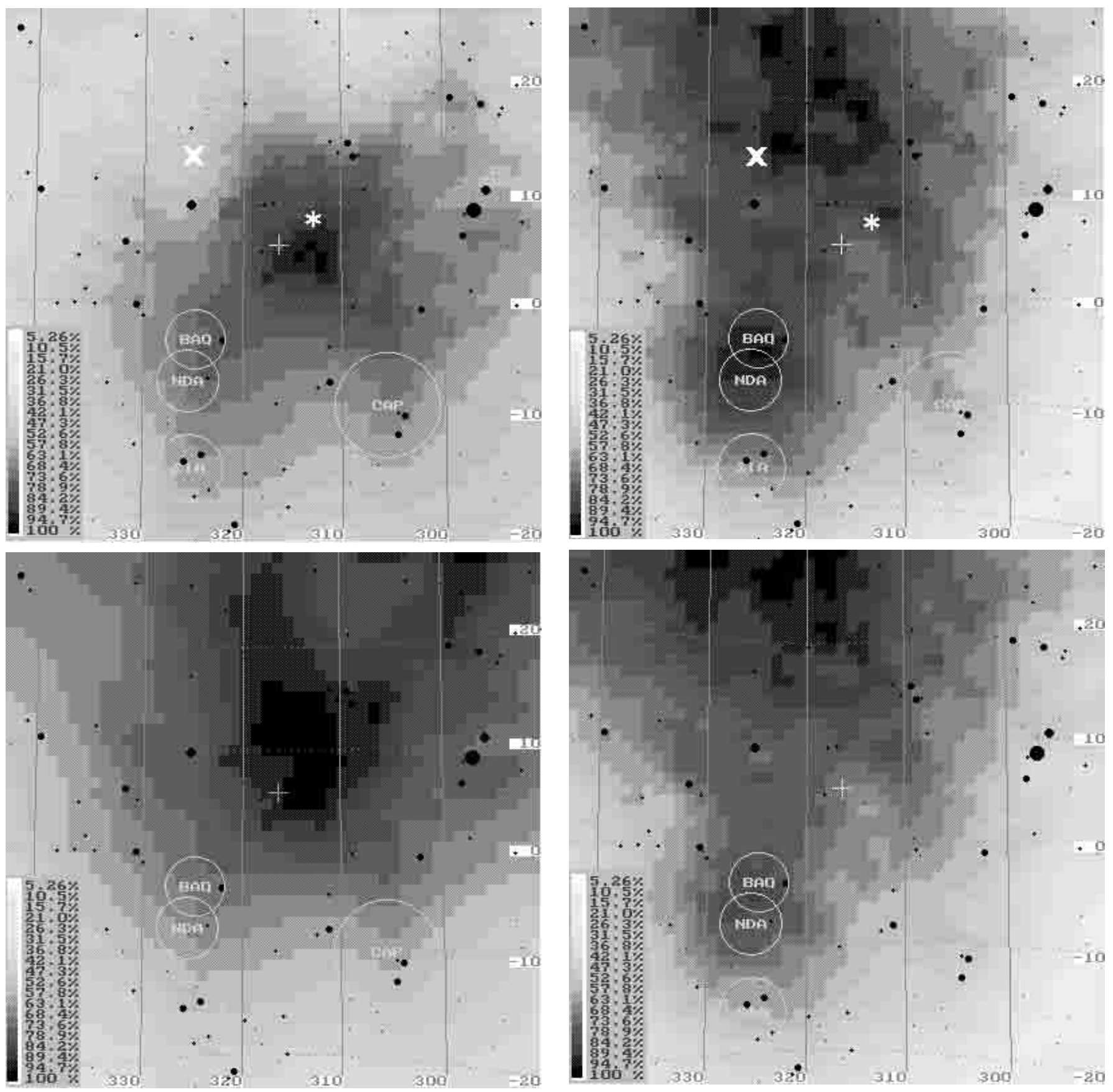

Fig. 13. PPR maps for a real sample of 6468 meteors observed in the years 1996-1999. All maps are computed for the following parameters: $\lambda_{\odot}=125^{\circ}, \Delta \lambda=1.0^{\circ}$ and $V_{\infty}=35 \mathrm{~km} \mathrm{~s}^{-1}$. The maximum distance of the meteor from the radiant is 50 and $85^{\circ}$, respectively from the upper to lower panel. The big cross marks the position of antihelion source and the asterisk the mean position of Delpninids' radiant derived from real observations of $C M W$.

computed for meteors placed within 50 degrees from the center of the map, suffer less from the meteors radiating from other showers and sources, we focus our discussion on them.

In the upper panel of Fig. 15 (we do not mark the position of the Delphinids' radiant for clarity) we detect a clear

Fig. 14. PPR maps for an artificial sample of 20376 meteors observed in the period July 8-31. All maps are computed for the following parameters: $\lambda_{\odot}=125^{\circ}, \Delta \lambda=1.0^{\circ}$ and $V_{\infty}=35 \mathrm{~km} \mathrm{~s}^{-1}$. The maximum distance of the meteor from the radiant is 50 and $85^{\circ}$, respectively from the upper to lower panel. The big cross marks the position of antihelion source and the asterisk the mean position of Delpninids' radiant derived from real observations of $C M W$.

clump of bright pixels at the Delphinids' radiant. They correspond to $15-18$ intersections suggesting the presence of the real radiant.

In the tracings map obtained from the simulated sample the strongest traces, indicating a high number of intersections, come from the real radiants of the Aquarid 

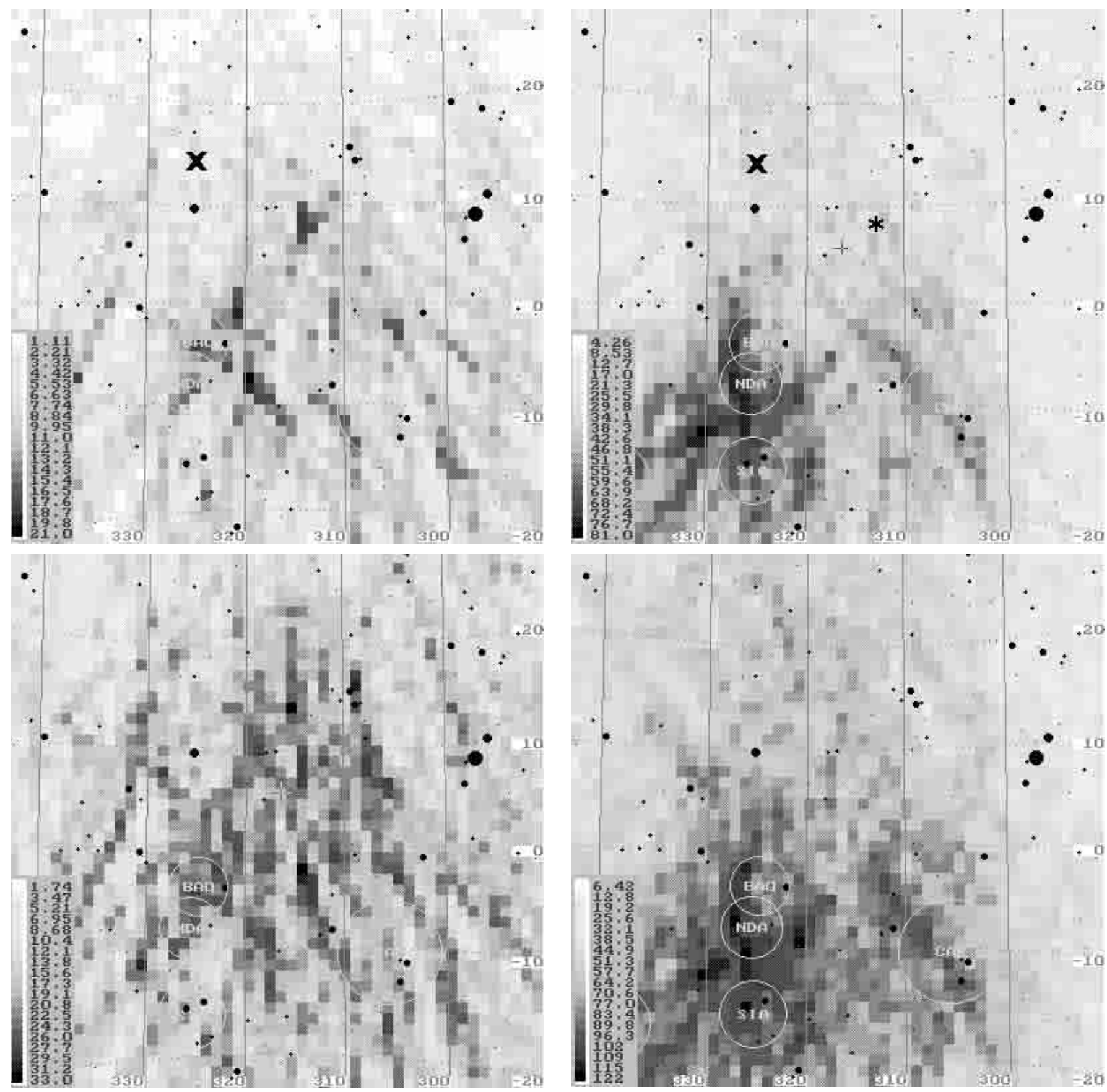

Fig. 15. Tracing maps for a real sample of 6468 meteors observed in the years 1996-1999. All maps are computed for the following parameters: $\lambda_{\odot}=125^{\circ}, \Delta \lambda=1.0^{\circ}$ and $V_{\infty}=$ $35 \mathrm{~km} \mathrm{~s}^{-1}$. The maximum distance of the meteor from the radiant is 50 and $85^{\circ}$, respectively from the upper to lower panel. The big cross marks the position of antihelion source.

complex. We also detect a wide and significantly less clear tail elongated toward the antihelion source. This tail reaches to the position of the radiant of the Delphinids shower and at this location shows about 30-40 intersections. It is less than half of the number of intersections detected in the area around the radiant of the Aquarid complex. In the case of the real sample, the trace of the Delphinids radiant was at the same level as the Aquarids

Fig. 16. Tracing maps for an artificial sample of 20376 meteors. All maps are computed for the following parameters: $\lambda_{\odot}=125^{\circ}, \Delta \lambda=1.0^{\circ}$ and $V_{\infty}=35 \mathrm{~km} \mathrm{~s}^{-1}$. The maximum distance of the meteor from the radiant is 50 and $85^{\circ}$, respectively from the upper to lower panel. The big cross marks the position of antihelion source and the asterisk the mean position of Delpninids' radiant derived from real observations of $C M W$.

and therefore we conclude that the tracings maps also indicate that the Delphinids are a real shower.

\section{Discussion}

We have compared two samples containing meteors observed in July in years 1996-1999. In the real sample, 


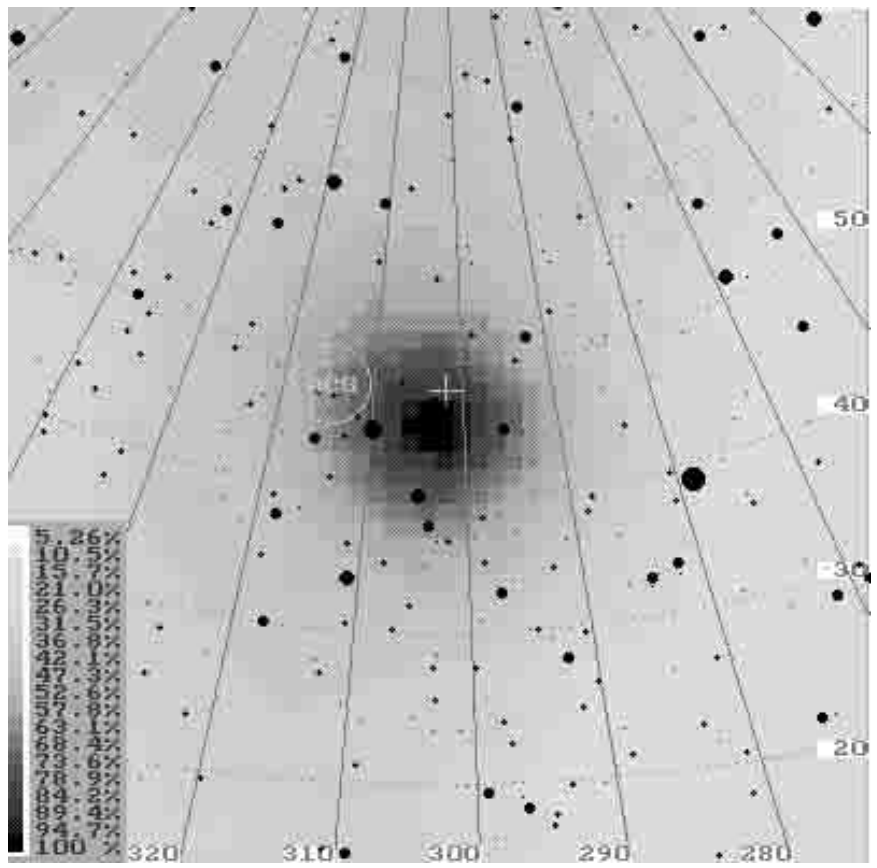

Fig. 17. The PPR map for an artificial sample of 24341 meteors with the $\alpha$-Cygnids and the Delphinids included and computed for the following parameters: $\lambda_{\odot}=115^{\circ}, \Delta \lambda=1.0^{\circ}$ and $V_{\infty}=41 \mathrm{~km} \mathrm{~s}^{-1}$. The maximum distance of the meteor from the radiant is $70^{\circ}$.

obtained from real visual observations made by Polish amateur astronomers, we detected clear radiants of $\alpha$-Cygnid and Delphinid showers. The question that we wanted to answer was whether these radiants can be produced as the intersections of paths of meteors radiating from the real showers active in July. Thus we constructed the artificial sample resembling in all details the real observations and we included all meteor showers except the $\alpha$-Cygnids and the Delphinids. These radiants, assuming that they are artificial formations created by intersections of meteors from real showers, should also be seen in the simulated sample. A comparison of both databases showed that it is very difficult to produce circular and clear radiants of the $\alpha$-Cygnids and the Delphinids using the meteors from an artificial sample. On the other hand such radiants are easy detected in the real sample. This strongly supports the hypothesis that the $\alpha$-Cygnids and the Delphinids indeed exist.

Finally, we decided to perform another test. To the artificial sample we added the meteors from the $\alpha$-Cygnids and the Delphinids. These showers were described by the parameters $Z H R_{\max }, B$ and $\lambda_{\odot(\max )}$ given by Stelmach \& Olech (2000) and Wiśniewski \& Olech (2001). The numbers of our artificial sample were then increased by $2035 \alpha$ Cygnids and 704 Delphinids.

We calculated the PPR maps for this new database and they are presented in Figs. 17 and 18 (for simplicity we decided to compute the maps for meteors within 70 degrees from the radiant only). Assuming that there are no other showers in July than the Delphinids, $\alpha$-Cygnids and

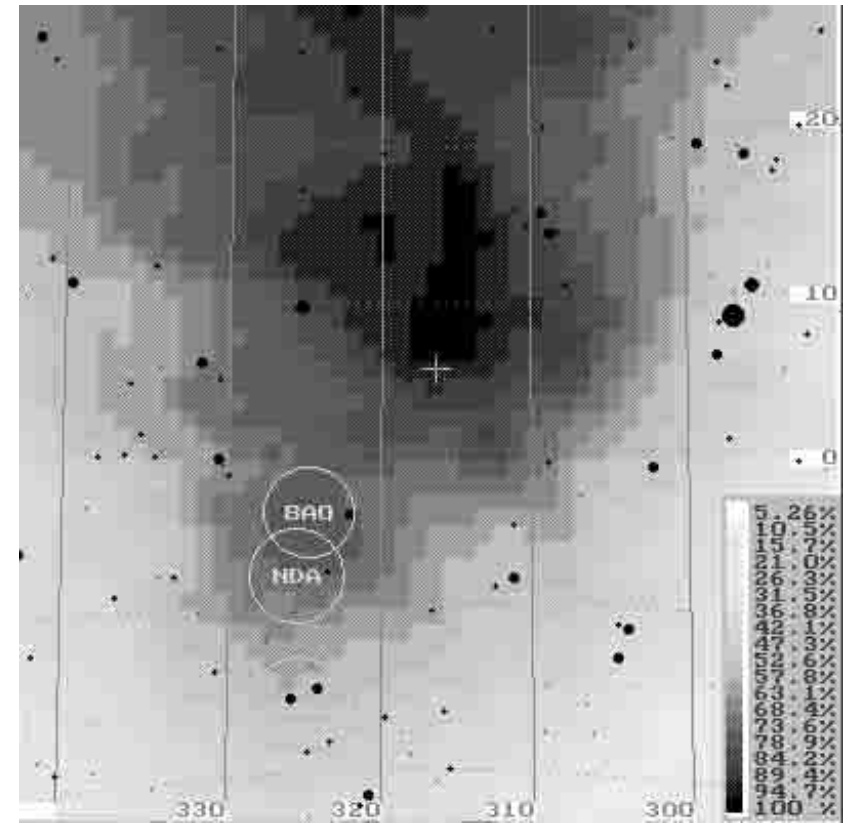

Fig. 18. The PPR map for an artificial sample of 23076 meteors with the $\alpha$-Cygnids and the Delphinids included and computed for the following parameters: $\lambda_{\odot}=125^{\circ}, \Delta \lambda=1.0^{\circ}$ and $V_{\infty}=35 \mathrm{~km} \mathrm{~s}^{-1}$. The maximum distance of the meteor from the radiant is $70^{\circ}$.

these listed in Table 1 we expect that our artificial sample should produce the same PPR maps as the real sample (shown in Figs. 9 and 13).

In the case of the $\alpha$-Cygnids we see that the artificial radiant is more compact than the one obtained from the real sample. However we should expect that there are few poorly known or even unknown showers which are present in the real sample and which were not included in the artificial sample. A good example is the $o$-Draconids shower, which is not listed in the IMO Working List of Meteor Showers, and as it is clearly visible from Fig. 9 is detected in our visual data causing a strong disturbance into the shape of the $\alpha$-Cygnids radiant.

In the case of the Delphinids both Fig. 18 and especially lower panel of Fig. 13 are similar. The radiant of the Delphinid shower is elongated toward the $\alpha$-Cygnids radiant. Also the influence of the Aquarid complex is present in both cases.

The similarity of the PPR maps obtained from the new artificial and real samples is another argument for the existence of the $\alpha$-Cygnids and the Delphinids.

Our artificial databases are accessible via the Internet and can be downloaded from the following URL: http://www.astrouw.edu.pl/ olech/SIM/. Detailed information about these databases are included in the README file.

Acknowledgements. We are grateful to Chris O'Connor and Dr. Marcin Kiraga for reading and commenting on this manuscript. This work was supported by KBN grant number 5 P03D 020 26. AO also acknowledges support from Fundacja 
na Rzecz Nauki Polskiej. Additionaly we would like to thank Prof. Marcin Kubiak from Warsaw University Observatory for allowing us to organize the $C M W$ astronomical camps in Ostrowik where the majority of our data was collected.

\section{References}

Abalakin, V. K. 1981, Astronomical Calendar. Ivariable Part. (Nauka. Moscow, in Russian)

Arlt, R. 1992, WGN, 20, 62

Arlt, R. 1997, WGN, Report Ser., 9

Arlt, R. 1998, WGN, Report Ser., 10

Arlt, R. 1999, WGN, Report Ser., 11

Arlt, R. 2000, WGN, Report Ser., 12

Babadzhanov, P. B., \& Kramer, E. N. 1961, Smits. Contr. Astrophys., 11, 67

Brown, P., \& Jones, J. 1995, Earth, Moon Planets, 68, 223

Denning, W. F. 1919, J. British. Astron. Assoc., 29, 161

Hawkins, G. S. 1957, MNRAS, 116, 92

Jenniskens, P. 1994, A\&A, 287, 990

Jones, J., \& Brown, P. 1993, MNRAS, 265, 524

Koschack, R. 1991, WGN, 19, 225

Kosinski, J. W. 1990, VI Zestawienie Rojów Meteorowych. AstroGeo, Wyszków, in Polish

Molau, S. 2000, Proc. International Meteor Conference Frasso Sabino, Italy, ed. R. Arlt, 31
Molau, S. 2001, Proc. International Meteor Conference Pucioasa, Romania, ed. R. Arlt, M. Triglav, \& C. Trainer, 52

Olech, A., Gajos, M., \& Jurek, M. 1999a, A\&AS, 135, 291

Olech, A., Jurek, M., \& Gajos, M. 1999b, Proc. International Meteor Conference Stárá Lesná, Slovakia, ed. R. Arlt, \& A. Knoefel, 58

Poole, L. M. G. 1997, MNRAS, 290, 245

Rendtel, J., Arlt, R., Koschack, R., et al. 1995, Meteor Shower Descriptions in Handbook for Visual Meteor Observers, ed. R. Arlt, \& A. McBeath (IMO, Potsdam), 126

Stelmach, D., \& Olech, A. 2000, Proc. International Meteor Conference Frasso Sabino, Italy, ed. R. Arlt, 71

Triglav, M. 2001, Proc. International Meteor Conference Pucioasa, Romania, ed. R. Arlt, M. Triglav, \& C. Trainer, 108

Velkov, V. 1996, Proc. International Meteor Conference Apeldoorn, the Netherlands, ed. A. Konoefel, P. Roggemans, 32

Wiśniewski, M., \& Olech, A. 2000, Proc. International Meteor Conference Frasso Sabino, Italy, ed. R. Arlt, 87

Wiśniewski, M., \& Olech, A. 2001, Proc. International Meteor Conference Pucioasa, Romania, ed. R. Arlt, M. Triglav, \& C. Trainer, 116

Znojil, V. 1995, Sporadic Meteors in Handbook for Visual Meteor Observers, ed. R. Arlt, \& A. McBeath (IMO, Potsdam), 110 